\title{
Protective Immunity against COVID-19
}

\author{
Anjali Priyadarshini*, Archana Gupta*, Manoj Kumar Yadav, Arpana Vibhuti, \\ Ramendra Pati Pandey, V. Samuel Raj \\ Centre for Drug Design Discovery and Development (C4D), SRM University, Delhi-NCR, \\ Rajiv Gandhi Education City, Sonepat - 131 029, Haryana, India
}

\section{*Shared co-first authorship}

\begin{abstract}
:
Tuberculosis and Covid-19 infection measure two quite different diseases- TB is caused by a sort of bacterium whereas Covid-19 is caused by a virus. However, the BCG immunizing agent would possibly facilitate individuals build immune responses to things aside from TB, inflicting "off-target effects," In different words, in run format, individuals started learning positive in obtaining the immunizing agent that had nothing to try and do with TB, several studies showed however the BCG immunizing agent affects individuals with kind one although the precise mechanism for these off-target effects of the BCG immunizing agent is not clear, it's believed that the immunizing agent will cause a nonspecific boost of the reaction. There is presently no immunizing agent or treatments approved by the United States of America Food and Drug Administration for the novel coronavirus. BCG is usually innocuous with the most facet impact the event of inflammation at the positioning of injection. Supported by these observations BCG so emerges as a possible candidate for the development of innate and adjustive reactions which can be non-specifically taking care of mycobacterium and different infectious agents against that vaccine remains not on the market.
\end{abstract}

Keywords: Tuberculosis, Covid-19, BCG, Immunizing agent, mycobacterium, infectious agents 


\section{Introduction:}

Other than SARS-CoV-2, there are six known coronaviruses that can infect humans: HCoV229E, HCoV-OC43, SARS-CoV, HCoVNL63, HCoV-HKU1, and MERS-CoV [1]. Still, we do not have any therapeutic measure against coronavirus causing COVID-19. Drug and vaccine development are a long process, and the mutation rate of this virus is very fast, so by the time we are ready with drugs and vaccines it may prove to be futile. The need of the time is to look for alternatives to tackle this menace. We need to equip our immune system well in advance to fight this deadly virus. There are vaccines available for other infectious diseases which bring about the activation of various cells thus arming the immune system against infection. These activated cells/pathways may act in a specific or non- specific manner. Viral infection elicits certain immune responses, we need to look for agents that can bring similar kinds of response and BCG seems to be a strong candidate for this purpose. Inoculation of the BCG vaccine by intradermal injection leads to the development of a site where [2,3] present in the vaccine.

BCG vaccine also known as pathogen-associated molecular patterns (PAMPs), are recognized by different pattern recognition receptors (PRRs) on the immune cells [2]. Toll-like receptors (TLRs) particularly TLR2 and TLR4 present on the cell surface membrane of immune cells are involved in BCG recognition [2]. The mycobacteria express a plethora of proteins, TLR agonists, which can stimulate the expression of macrophages and DCs resulting in the secretion of proinflammatory cytokines [4]. Aside from this, another method of acknowledgment is the association of supplement receptors CR3 and CR4 which encourage DCs to perceive opsonized mycobacteria. Another gathering of cell receptor that perceive BCG PAMPs are nucleotiderestricting oligomerization space (NOD)- like receptors found in the cytosol of inborn resistant cells, for example, NOD2, which associate with a particular segment of the bacterial peptidoglycan [5]. In addition, C-type lectins, for example, DC-explicit intercellular grip atom 3getting non-integrin (DC-SIGN) associate with parts of the bacterial divider and are associated with the acknowledgment and disguise of BCG [5]. After disguise by DCs, the mycobacterium can satisfy fourteen days inside these cells [6]. This communication prompts DC development and movement that is described by an expansion in the declaration of co-stimulatory atoms, for example, CD40, CD80, CD83, and CD86 [7]. One of the antigens present on the cell mass of BCG compares to an antigen (Ag) 85 (additionally present in M. tuberculosis), which invigorates the creation of tumour rot factor-alpha (TNF-a), interleukin 1-beta (IL-1b), and IL-6 [8], which 
can produce a proinflammatory express that advances the initiation of resistant cells. This marvel related with a memory-like reaction in natural resistant cells is known as "prepared invulnerability."

It has been suggested that the nonspecific disease resistance induced by BCG is mediated by an enhanced memory-like response of the innate immune system known as 'trained' immunity [9]. Trained immunity is induced primarily in myeloid cells (monocytes and macrophages) and natural killer (NK) cells [10], after previous exposure to some live vaccines like BCG, measles, and yellow fever, as well as to some microbial components of pathogens [11]. Various studies have demonstrated that trained immunity is independent of adaptive immunity [12], and is caused by epigenetic reprogramming and alterations in basal intracellular metabolic pathways, resulting in changes in gene expression and cell physiology. These changes lead to increased innate immune cells' capacity to respond to stimulation [13]. Change in methylation designs in specific qualities in coursing monocytes can prompt the articulation or potentially suppression of qualities. This may eventually prompt expanded security against optional diseases after inoculation, because of improved microbe acknowledgment and quicker fiery reactions. It has been proposed the idea of "prepared invulnerability," which is characterized as an expanded vague reaction to an optional disease interceded by the inborn resistant framework, either to the equivalent or various microorganisms. This sort of invulnerability is portrayed as being autonomous of $\mathrm{T}$ and $\mathrm{B}$ cell reactions and is interceded by monocytes/macrophages and NK cells. With respect to the instance of the flu an infection, the high change pace of this microorganism can debilitate the viability of exceptionally explicit immunizations, accordingly the wide range of microbes to which prepared resistance reaction could be an extraordinary bit of leeway for the assurance of such irresistible specialists [14,15]. Moreover, popular contaminations, for example, RSV have been appeared to build the host weakness to bacterial diseases [16].

\section{BCG vaccines protect from Type-I diabetes:}

In the world Several BCG vaccines, based on different strains, are available worldwide helps in providing protective immunity against $M$. tuberculosis to infants, and thus translate into improved protection against TB.BCG vaccine that is primarily developed to combat tuberculosis also confers non- specific protection against other neonatal diseases. In the bladder cancer, 
intravascular BCG boosts host immunity, reduces tumor recurrence progression and decreases mortality, and has been approved for use in bladder cancer since the 1990s. It is well evident that BCG helps in improving non-specific immunity against listeria and influenza in the murine model. Inoculation with BCG strain forces an individual's innate immune system to develop trained memory, through epigenetic reprogramming of different innate immune cell [17]. Researchers de-mystify the molecular mechanism behind the success of BCG vaccination in a plethora of diseases. The vaccination tempts viral immunity. Thus, we are interested to check the extended role of BCG vaccination in the reduction of COVID-19 incidences. Global comparison of the trajectory of the COVID-19 outbreak shows the prominent and distinct differences in morbidity and mortality rates across the world. The region-wise availability of COVID-19 data on different platforms shows a clear bifurcation in the trajectory of the outbreak. One group of countries (Brazil, Germany, Italy, Iran, South Korea, Spain, US, etc.). Is there any factor that provides comparative immunity against COVID-19 missing in the first group? COVID 19 mortality is remarkably high in countries like the United States, Italy, Spain where the BCG vaccination program is never implemented. On the other hand, countries like Japan had a low mortality rate without implementing any strictest. While Iran had started giving BCG vaccination only in around, they were heavily hit by COVID-19. Currently, however, the situation in China seems to be improved. Consistent with the idea that protecting the elderly population might be crucial in reducing mortality [18]. Although the number of cases is low in countries having an early implementation of BCG programs, the differential rate of COVID-19 related incidences and deaths poses a doubt about this relationship. One explanation is the use of three different BCG strains that confer differential immunity in different regions of the world. Studies on the efficacy of BCG vaccination indicate a significant difference in immune responses induced in the newborn by different BCG strains. This is one of the possible reasons for low mortality in countries like Japan where BCG vaccination was used.

\section{Correlation of BCG Vaccination with COVID-19:}

The BCG World Atlas [19] divides countries with information on BCG vaccination into 3 groups. The second group consists of countries where universal BCG vaccination policies existed in the past but are no longer active, colour coded with violet. The third group consists of countries where BCG vaccination is not universal. This group is colour coded with dark green. 
Canada and the US are the two members of this group. Details of the variation in BCG vaccination across the 20 countries are given in the table table-1. Australia and Spain stopped the vaccination programs in the early 1980s; Germany, which had a universal program, stopped it in the late 1990s; Italy did not have a universal. The USA has reported almost 5,331,15 cases with more than 20,500 deaths, Italy has 1,50,000 cases, and over 19,000 fatalities.

It is too early to form any final conclusion with BCG vaccination with a range of COVID-19, Researchers can have to be compelled to fastidiously rule out the chance of a correlation by dominant for alternative contradictory factors, like country-specific public health interventions, which may be driving the determined pattern. They are going to conjointly must be compelled to determine a plausible causative mechanism that may link the BCG vaccination to COVID-19. If there is any truth in these patterns, Bharat would possibly see a comparatively less devastating natural event of COVID-19 than several Western European and North Yankee countries because of it adopted universal BCG vaccination in 1948.

Table 1: BCG Vaccination across countries

\begin{tabular}{|c|c|c|c|}
\hline $\begin{array}{c}\text { BCG Vaccination across } \\
\text { countries }\end{array}$ & $\begin{array}{c}\text { BCG } \\
\text { Vaccination } \\
\text { starts }\end{array}$ & $\begin{array}{c}\text { BCG Vaccination } \\
\text { ends }\end{array}$ & Universal \\
\hline Australia & 1950 & 1985 & Yes \\
\hline Bangladesh & 1979 & Ongoing & Yes \\
\hline Brazil & 1976 & Ongoing & Yes \\
\hline Czechia & 1953 & 2010 & Yes \\
\hline Germany & 1961 & 1998 & Yes \\
\hline India & 1948 & Ongoing & Yes \\
\hline Indonesia & 1999 & Ongoing & Yes \\
\hline Iran & 1984 & Ongoing & Yes \\
\hline Italy & 1970 & 2001 & No \\
\hline Japan & 1951 & Ongoing & Yes \\
\hline Kenya & Not known & Ongoing & Yes \\
\hline Malaysia & Not known & Ongoing & Yes \\
\hline Mexico & 1951 & Ongoing & Yes \\
\hline Nigeria & 1991 & Ongoing & Yes \\
\hline Pakistan & 1978 & Ongoing & Yes \\
\hline South Korea & 1970 & Ongoing & Yes \\
\hline Spain & 1965 & Ongoing & Yes \\
\hline Sri Lanka & Not known & Ongos \\
\hline Thailand & 1977 & Ongoing & Yes \\
\hline USA & None & None & No \\
\hline
\end{tabular}




\section{Conclusions:}

The countries affected by malaria show that it inversely correlates with the number of COVID 19 deaths. In other words, there is a clear basis to hypothesize a potential link between anti-malaria prophylaxis and the prevention of the novel coronavirus infection. Chloroquine and its analogs are an effective preventive treatment for malaria [20] indicated, chloroquine seems to have a strong antiviral effect on SARS-CoV several trials conducted in China on the Covid-19 have also shown that chloroquine and hydroxychloroquine improved the symptomatology and the outcome of the infection. It is important to further investigate the use of chloroquine-based medications as a preventative intervention to halt the spread of the COVID-19 infection, and the possibility of a massive prevention/early-intervention campaign with the wide distribution of chloroquine to those at risk of infection, or already infected. As the infectious disease malaria is considered to be a threat in 91 countries and territories, and it affects mainly Africa followed by South East Asia and the eastern Mediterranean region, it is important to study the SASR CoV-2 cases in these countries. These countries are administered routinely antimalarial drugs like chloroquine and hydroxychloroquine. As shown in the table the numbers of COVID-19 cases are less as compared to developed countries so the major question is to find out the possible correlation between the countries having high malaria rates (where the antimalarial drugs are routinely administered) and lower cases of COVID-19. As this is not just a simple coincidence, this correlation needs to be explored further. Moreover, chloroquine is likely to have both prophylactic and therapeutic advantages. These drugs work by inhibiting the glycosylation process which is a chemical transformation of the proteins in the virus's outer shell that is part of the infection process. Recent in vitro studies showed that both hydroxychloroquine and chloroquine have antiviral properties against COVID-19.

\section{Consent for Publication:}

Not applicable

Funding:

None

\section{Conflict of Interest:}

The author declares no conflict of interest, financial or otherwise.

\section{Acknowledgements:}

Declared none

\section{References:}


[1]. Li Q, Guan X, Wu P, Wang X, Zhou L, Tong Y, Ren R, Leung KSM, Lau EHY, Wong JY, Xing X, Xiang N, Wu Y, Li C, Chen Q, Li D, Liu T, Zhao J, Liu M, Tu W, Chen C, Jin L, Yang R, Wang Q, Zhou S, Wang R, Liu H, Luo Y, Liu Y, Shao G, Li H, Tao Z, Yang Y, Deng Z, Liu B, Ma Z, Zhang Y, Shi G, Lam TTY, Wu JT, Gao GF, Cowling BJ, Yang B, Leung GM, Feng Z. Early Transmission Dynamics in Wuhan, China, of Novel Coronavirus-Infected Pneumonia. N Engl J Med. 2020 Mar 26;382(13):1199-1207.

[2]. Dockrell HM, Smith SG. What have we learnt about BCG vaccination in the last 20 years? Front Immunol. (2017) 8:1134. doi: 10.3389/fimmu.2017.01134

[3]. Moliva JI, Turner J, Torrelles JB. Immune responses to bacillus calmette-guérin vaccination: why do they fail to protect against Mycobacterium tuberculosis? Front Immunol. (2017) 8:407.

[4]. Kumar S, Sunagar R, Gosselin E. Bacterial protein toll-like-receptor agonists: a novel perspective on vaccine adjuvants. Front Immunol. (2019) 10:1144.

[5]. Gagliardi MC, Teloni R, Giannoni F, Pardini M, Sargentini V, Brunori L, et al. Mycobacterium bovis bacillus calmette-guerin infects DC-SIGNdendritic cell and causes the inhibition of IL-12 and the enhancement of IL-10 production. J Leukoc Biol. (2005) 78:106-13.

[6]. Jiao X, Lo-Man R, Guermonprez P, Fiette L, Dériaud E, Burgaud S, et al. Dendritic cells are host cells for mycobacteria in vivo that trigger innate and acquired immunity. J Immunol. (2002) 168:1294-301. doi: 10.4049/jimmunol.168.3.1294

[7]. Tsuji S, Matsumoto M, Takeuchi O, Akira S, Azuma I, Hayashi A, et al. Maturation of human dendritic cells by cell wall skeleton of Mycobacterium bovis bacillus calmette-guérin : involvement of toll-like receptors. Infect Immun. (2000) 68:6883-90. doi: 10.1128/IAI.68.12.6883-6890.2000

[8]. Netea MG, Joosten LA, Latz E, Mills KH, Natoli G, Stunnenberg HG, et al. Trained immunity: A program of innate immune memory in health and disease. Science. 2016; 352(6284).

[9]. Netea MG, Quintin J, Van Der Meer JWM. Trained immunity: a memory for innate host defense. Cell Host Microbe. (2011) 9:355-61. doi: 10.1016/j.chom.2011.04.006

[10]. Kleinnijenhuis J, Van Crevel R, NeteaMG. Trained immunity: consequences for the heterologous effects of BCG vaccination. Trans R Soc Trop Med Hyg. (2014) 109:29-35. doi: $10.1093 /$ trstmh/tru 168

[11]. Kaufmann E, Sanz J, Dunn JL, Khan N, Mendonça LE, Pacis A, et al. BCG educates hematopoietic stem cells to generate protective innate immunity against tuberculosis. Cell. (2018) 172:176-90. e19. doi: 10.1016/j.cell.2017.12.031 
[12]. Aaby P, Kollmann TR, B enn CS. Nonspecific effects of neonatal and infant vaccination: public-health, immunological and conceptual challenges. Nat Immunol. 2014; 15(10):895-9.

[13].Kleinnijenhuis, J., J. Quintin, F. Preijers, L. A. Joosten, D. C. Ifrim, S. Saeed, C. Jacobs, J. van Loenhout, D. de Jong, H. G. Stunnenberg, R. J. Xavier, J. W. van der Meer, R. van Crevel and M. G. Netea (2012). "Bacille Calmette-Guerin induces NOD2-dependent nonspecific protection from reinfection via epigenetic reprogramming of monocytes." Proc Natl Acad Sci U S A 109(43): 17537-17542.

[14].Xue KS, Moncla LH, Bedford T, Bloom JD. Within-host evolution of human influenza virus. Trends Microbiol. (2018) 26:781-93. doi: 10.1016/j.tim.2018.02.007

[15]. Harding AT, Heaton NS. Efforts to improve the seasonal influenza vaccine. Vaccines. (2018) 6:E19. doi: 10.3390/vaccines 6020019

[16].Nam HH, Ison MG. Respiratory syncytial virus infection in adults. BMJ. (2019) 366:15021.

[17].Arts, R. J. W., S. J. C. F. M. Moorlag, B. Novakovic, Y. Li, S.-Y. Wang, M. Oosting, V. Kumar, R. J. Xavier, C. Wijmenga, L. A. B. Joosten, C. B. E. M. Reusken, C. S. Benn, P. Aaby, M. P. Koopmans, H. G. Stunnenberg, R. van Crevel and M. G. Netea (2018). "BCG Vaccination Protects against Experimental Viral Infection in Humans through the Induction of Cytokines Associated with Trained Immunity." Cell Host \& Microbe 23(1): 89-100.e105.

[18].Shet, A., D. Ray, N. Malavige, M. Santosham and N. Bar-Zeev (2020). "Differential COVID-19-attributable mortality and BCG vaccine use in countries." medRxiv: 2020.2004.2001.20049478.

[19]. Zwerling, A., M. A. Behr, A. Verma, T. F. Brewer, D. Menzies and M. Pai (2011). "The BCG World Atlas: a database of global BCG vaccination policies and practices." PLoS medicine 8(3): e1001012-e1001012.

[20].Vincent MJ, Bergeron E, Benjannet S, Erickson BR, Rollin PE, Ksiazek TG, Seidah NG, Nichol ST. Chloroquine is a potent inhibitor of SARS coronavirus infection and spread. Virol J. 2005 Aug 22; 2:69. 